DIGITALCOMMONS

—@WAYNESTATE -
Journal of Modern Applied Statistical Methods

$11-1-2011$

\title{
Estimation and Hypothesis Testing in LAV Regression with Autocorrelated Errors: Is Correction for Autocorrelation Helpful?
}

Terry E. Dielman

Texas Christian University, t.dielman@tcu.edu

Follow this and additional works at: http://digitalcommons.wayne.edu/jmasm

Part of the Applied Statistics Commons, Social and Behavioral Sciences Commons, and the Statistical Theory Commons

\section{Recommended Citation}

Dielman, Terry E. (2011) "Estimation and Hypothesis Testing in LAV Regression with Autocorrelated Errors: Is Correction for Autocorrelation Helpful?," Journal of Modern Applied Statistical Methods: Vol. 10 : Iss. 2 , Article 13.

DOI: $10.22237 /$ jmasm/1320120720

Available at: http://digitalcommons.wayne.edu/jmasm/vol10/iss2/13

This Regular Article is brought to you for free and open access by the Open Access Journals at DigitalCommons@WayneState. It has been accepted for inclusion in Journal of Modern Applied Statistical Methods by an authorized editor of DigitalCommons@WayneState. 


\title{
Estimation and Hypothesis Testing in LAV Regression with Autocorrelated Errors: Is Correction for Autocorrelation Helpful?
}

\author{
Terry E. Dielman \\ Texas Christian University \\ Fort Worth, TX
}

Using the Prais-Winsten correction and adding a lagged variable provides improved estimates (smaller MSE) in least absolute value (LAV) regression when moderate to high levels of autocorrelation are present. When comparing empirical levels of significance for hypothesis tests, adding a lagged variable outperforms other approaches but has a relative high empirical level of significance.

Key words: Monte Carlo simulation, serial correlation, Cochrane-Orcutt, Prais-Winsten, lagged variable.

\section{Introduction}

Least absolute value (LAV) regression is one technique often suggested for robust regression (see Dielman, 2005 for a review of LAV research). LAV estimates are less strongly affected by extreme observations compared to their least squares counterparts. The use of regression to model time-series data often results in the violation of the assumption of independent disturbances. The Prais-Winsten (PW) and Cochrane-Orcutt (CO) methods are two procedures used for correcting for autocorrelation in time-series regression models: Both methods transform the data using a differencing transformation to remove autocorrelation. LAV estimation applied to the transformed observations yields estimators that are asymptotically more efficient than LAV applied to the original data. The two methods are essentially equivalent except for the treatment of the first observation in the data set. The CO method omits the first observation; the PW method transforms and retains the observation. Asymptotically, no difference exists in the

Terry E. Dielman is a Professor of Decision Sciences in the Information Systems and Supply Chain Management Department in the M. J. Neeley School of Business. Email him at: t.dielman@tcu.edu. efficiency of estimators produced by the two methods. In previous studies of small sample behavior, however, the PW procedure has been found to produce more efficient estimates; using the $\mathrm{CO}$ procedure results in estimators that can be much less efficient in small samples.

Koenker and Bassett (1982) suggested the WALD, likelihood ratio (LR), and Lagrange multiplier (LM) tests for coefficient significance when using LAV estimation. Stangenhaus (1987), Dielman and Pfaffenberger (1990, 1992), Dielman and Rose (1996), and Koenker (1987) have studied inference for regression using LAV estimation when disturbances are independent but not necessarily normal.

Some research has considered LAV estimation when errors are not independent. Dielman and Rose (1994a, 1995b) examined the accuracy of estimation for model coefficients using LAV regression with autocorrelation correction, and Dielman and Rose (1994b) considered the accuracy of forecasts from LAV estimated regressions with autocorrelation correction. Dielman and Rose (1997) examined both estimation and inference in autocorrelated models.

A simulation study was conducted to address questions of estimation and inference in the presence of serial correlation. The PW and $\mathrm{CO}$ corrections for autocorrelation are considered and compared to the performance of a model with a lagged dependent variable added. Estimation accuracy after correction for autocorrelation is compared using mean square 


\section{LAV REGRESSION WITH AUTOCORRELATED ERRORS: IS CORRECTION HELPFUL?}

estimation error. The performance of hypothesis tests for the slope coefficient is assessed using observed significance levels, and alternative estimators of the scale parameter used in the test procedures are considered. In addition, performance in small samples is considered due to the practical importance of smaller sample sizes - particularly for applications in business and economics - and the inability to rely upon asymptotic results under such circumstances.

Methodology

A simple regression model is considered:

with

$$
y_{t}=\beta_{0}+\beta_{1} x_{\mathrm{t}}+\varepsilon_{\mathrm{t}}
$$

$$
\varepsilon_{\mathrm{t}}=\rho \varepsilon_{\mathrm{t}-1}+\eta_{\mathrm{t}}
$$

for $t=1,2, \ldots, T$. In (1), $y_{t}$ and $x_{t}$ are the $\mathrm{t}^{\text {th }}$ observations on the dependent and explanatory variables, respectively, and $\varepsilon_{t}$ is a random disturbance for the $\mathrm{t}^{\text {th }}$ observation and may be subject to autocorrelation. The $\eta_{t}$ represents disturbance components that are assumed to be independent and identically distributed, although not necessarily normal. The parameters $\beta_{0}$ and $\beta_{1}$ are unknown and must be estimated. The parameter $\rho$ is the autocorrelation coefficient, with $|\rho|<1$.

Using matrix notation, the model can be written as:

$$
\mathbf{Y}=\mathbf{X} \boldsymbol{\beta}+\boldsymbol{\varepsilon}
$$

where

$$
\mathrm{Y}=\left[\begin{array}{c}
\mathrm{y}_{1} \\
\mathrm{y}_{2} \\
\cdot \\
\cdot \\
\cdot \\
\mathrm{y}_{\mathrm{T}}
\end{array}\right], \mathrm{X}=\left[\begin{array}{cc}
1 & \mathrm{x}_{1} \\
1 & \mathrm{x}_{2} \\
\cdot & \cdot \\
\cdot & \cdot \\
\cdot & \cdot \\
1 & \mathrm{x}_{\mathrm{T}}
\end{array}\right], \varepsilon=\left[\begin{array}{c}
\varepsilon_{1} \\
\varepsilon_{2} \\
\cdot \\
\cdot \\
\cdot \\
\varepsilon_{\mathrm{T}}
\end{array}\right], \beta=\left[\begin{array}{c}
\beta_{0} \\
\beta_{1}
\end{array}\right] .
$$

Two well-known procedures employed to correct for autocorrelation are the Prais-
Winsten (1954) and Cochrane-Orcutt (1949) procedures. Both transform the data using the autocorrelation coefficient, $\rho$, after which the transformed data are used in estimation. The procedures differ in their treatment of the first observation, $\left(x_{1}, y_{1}\right)$. The $\mathrm{PW}$ transformation matrix is:

$$
\mathrm{M}_{\mathrm{PW}}=\left[\begin{array}{ccccccc}
\left(1-\rho^{2}\right)^{1 / 2} & 0 & . & . & . & 0 & 0 \\
-\rho & 1 & . & . & . & 0 & 0 \\
\cdot & . & . & & & . & . \\
\cdot & . & & . & & . & . \\
. & . & & & . & & \\
0 & 0 & . & . & . & -\rho & 1
\end{array}\right] .
$$

Pre-multiplying the model in (2) by $\mathbf{M}_{\mathrm{PW}}$ yields

$$
\mathrm{M}_{\mathrm{PW}} \mathrm{Y}=\mathrm{M}_{\mathrm{PW}} \mathrm{X} \beta+\mathrm{M}_{\mathrm{PW}} \varepsilon
$$

or

$$
\mathbf{Y}^{*}=\mathbf{X}^{*} \boldsymbol{\beta}+\boldsymbol{\eta}
$$

where $\mathbf{Y}^{*}$ contains the transformed dependent variable values and $\mathbf{X}^{*}$ is the matrix of transformed independent variable values, thus:

$$
\mathbf{Y}^{*}=\left\lfloor\left(1-\rho^{2}\right)^{1 / 2} y_{1}, y_{2}-\rho y_{1}, \ldots, y_{T}-\rho y_{T-1}\right\rfloor
$$

and

$$
\mathbf{X}^{*}=\left[\begin{array}{cc}
\left(1-\rho^{2}\right)^{1 / 2} & \left(1-\rho^{2}\right)^{1 / 2} x_{1} \\
1-\rho & x_{2}-\rho x_{1} \\
\cdot & \cdot \\
\cdot & \cdot \\
\cdot & \cdot \\
1-\rho & x_{T}-\rho x_{T-1}
\end{array}\right]
$$

In (6), $\boldsymbol{\eta}$ is the vector of serially uncorrelated $\eta_{t}$ errors.

The CO transformation matrix is the $(T-1) \times 1$ matrix obtained by removing the first row of the $\mathbf{M}_{\mathbf{P w}}$ transformation matrix. The use of the $\mathrm{CO}$ transformation means that $(T-1)$ observations, rather than $T$, are used to estimate the model. In the $\mathrm{CO}$ transformation, the first 


\section{TERRY E. DIELMAN}

observation is omitted, whereas it is transformed and included in the estimation in the PW transformation. Asymptotically, the loss of this single observation is of minimal concern. However, for small samples, omitting the first observation may result in an estimator inferior to that obtained when the first observation is retained and transformed as shown in Maeshiro (1979), Park and Mitchell (1980) and Dielman and Pfaffenberger (1984) for least squares and in Dielman and Rose (1994a) for LAV. The two methods described are referred to as LAVPW and LAVCO when combined with LAV estimation.

In practice, the value of $\rho$ will be unknown. In this case it must be estimated from sample data. The estimator of $\rho$ is as follows:

$$
\hat{\rho}_{P W}=\frac{\sum_{t=2}^{T} \hat{\varepsilon}_{t} \hat{\varepsilon}_{t-1}}{\sum_{t=2}^{T} \hat{\varepsilon}_{t}^{2}}
$$

when PW correction is used, and

$$
\hat{\rho}_{C O}=\frac{\sum_{t=2}^{T} \hat{\varepsilon}_{t} \hat{\varepsilon}_{t-1}}{\sum_{t=1}^{T-1} \hat{\varepsilon}_{t}^{2}}
$$

when $\mathrm{CO}$ correction is used, where $\hat{\varepsilon}_{t}$ represents LAV residuals from the uncorrected LAV regression. These are the estimators suggested by Park and Mitchell (1980) when using least squares estimation and are also typical of those that have been used in the LAV context.

An alternative approach suggested by Mizon (1995) is to include a lagged dependent variable as an explanatory variable and view this as part of the data generating process (DGP). No other testing for autocorrelation or correction for autocorrelation would be used. The model suggested can be written

$$
y_{t}=\beta_{0}+\beta_{1} x_{\mathrm{t}}+\beta_{2} y_{\mathrm{t}-1}+\eta_{\mathrm{t}},
$$

for $t=2, \ldots, T$ (note that $\mathrm{t}=1$ is not used due to the inclusion of the lagged variable). In (11), $y_{t}$ and $x_{t}$ are the $\mathrm{t}^{\text {th }}$ observations on the dependent and explanatory variables, respectively. The $\eta_{t}$ represents disturbance components, which are assumed to be independent and identically distributed, although not necessarily normal. The parameters $\beta_{0}, \beta_{1}$ and $\beta_{2}$ are unknown and must be estimated; however, in this application it is $\beta_{1}$ that is of interest. This method is referred to as LAVLAG.

Referring to the model in (2), Bassett and Koenker (1978) showed that the LAV coefficient estimator has an asymptotic distribution that converges to $N\left(\boldsymbol{\beta}, \lambda^{2}\left(\mathbf{X}^{\prime} \mathbf{X}\right)^{-1}\right)$ where $\frac{\lambda^{2}}{T}$ is the asymptotic variance of the sample median for a sample of size $T$ from the disturbance distribution. The scale parameter, $\lambda$, is defined as $\lambda=1 /[2 f(m)]$, where $f(m)$ is the probability density function (pdf) of the disturbance distribution evaluated at the median. These same results are obtained when $\mathbf{X}$ is replaced by $\mathbf{X}^{*}$ for the model in (6) (Weiss, 1990).

The test considered in this study is the basic test for slope coefficient significance, i.e., $\mathrm{H}_{0}: \beta_{1}=0$.

Three test statistics were examined: the WALD, the Likelihood Ratio (LR) and the Lagrange Multiplier (LM). The WALD, LR and LM statistics each have, asymptotically, a Chisquare distribution with $k_{2}$ degrees of freedom. (See Koenker and Bassett (1982) for further details on these test statistics.) The small sample properties of the test statistics are analytically intractable. Examination of the empirical level of significance of the test statistics in small samples was performed using a simulation.

Both the WALD and LR test statistics require the estimation of the scale parameter $\lambda$, whereas the LM test statistic does not. One often-suggested estimator for $\lambda$ can be computed as follows:

$$
\hat{\lambda}=\frac{\left(T^{\prime}\right)^{1 / 2}\left[e_{\left(T^{\prime}-m-1\right)}-e_{(m)}\right]}{z_{\alpha / 2}}
$$

where

$$
m=\frac{T^{\prime}+1}{2}-z_{\alpha / 2}\left(\frac{T^{\prime}}{4}\right)^{1 / 2}
$$




\section{LAV REGRESSION WITH AUTOCORRELATED ERRORS: IS CORRECTION HELPFUL?}

where the $e_{(.)}$are ordered residuals from the LAV-fitted model, and $T^{\prime}=T-r$ where $r$ is the number of zero residuals. A value of $\alpha=0.05$ is typically suggested. This estimator is referred to as the SECI estimator. McKean and Schrader (1984) used Monte Carlo simulation to compare several methods of studentizing the sample median in which the SECI performed well and the value of $\alpha=0.05$ produced the best results.

Sheather (1987) summarized the results of a Monte Carlo simulation to compare the SECI estimator and several other estimators for $\lambda$, including some that do not extend easily to the regression application. The conclusion was that the SECI estimator provides a good, quick point estimate of the standard error. Dielman and Pfaffenberger (1992) and Dielman and Rose (1996) also noted that this estimator performs reasonably well when used to compute the LR test statistic.

In this study, four different options in constructing the estimator of $\lambda$ were considered. as follows:

1. SECI1: $\hat{\lambda}_{1}$ uses $z=1.96$ (the $\alpha=0.05$ value) and $T^{\prime}=$ total number of observations $(T)$.

2. SECI2: $\hat{\lambda}_{2}$ uses $\mathrm{t}_{0.025}$ with $T$ degrees of freedom rather than the $\mathrm{z}$ value and $T^{\prime}=$ total number of observations $(T)$.

3. SECI3: $\hat{\lambda}_{3}$ uses $z=1.96$ (the $\alpha=0.05$ value) and $T^{\prime}=T-r$ where $r$ is the number of zero residuals.

4. SECI4: $\hat{\lambda}_{4}$ uses $t_{0.025}$ with $T-r$ degrees of freedom rather than the $\mathrm{z}$ value and $T^{\prime}=T$ $-r$ where $r$ is the number of zero residuals.

The notation $\mathrm{W} 1, \mathrm{~W} 2, \mathrm{~W} 3$ and $\mathrm{W} 4$ is used to indicate the WALD test using variance estimator 1, 2, 3 or 4, and L1, L2, L3 and L4 indicate the LR test using variance estimator 1, 2, 3 or 4. Most literature in this area recommends using the estimator SECI3. These options were considered in Dielman (2006) for models with independent errors and SECI1 and SECI2 were found to produce improved results over SECI3 in small samples. As noted, the LM test does not require the use of an estimate of $\lambda$.

The model considered in this study is described in (1). The explanatory variable values were generated as follows:

1. Autoregressive independent variable: $x_{\mathrm{t}}=$ $a x_{\mathrm{t}-1}+u_{\mathrm{t}}$ for $t=1,2, \ldots, T$ with $u_{\mathrm{t}}$ chosen from the $\mathrm{N}(0,2)$ distribution. The values of $a$ used were $0.0,0.4$ and 0.8

2. Stochastic trend: $x_{\mathrm{t}}=a t+u_{\mathrm{t}}$ for $t=1,2, \ldots, T$ with the $u_{\mathrm{t}}$ chosen from the $\mathrm{N}(0,2)$ distribution. The values of $a$ used were 0.4 and 0.8 .

3. Linear time trend: $x_{t}=t$ for $t=1,2, \ldots ., T$

After being generated, the independent variable values are held fixed throughout the experiment. The disturbances, $\eta_{t}$, were chosen from one of the following disturbance distributions:

1. Normal $(0,1)$;

2. Laplace with mean 0 and variance 2 ;

3. Contaminated normal with disturbances drawn from the standard normal distribution $85 \%$ of the time, and a normal distribution with mean 0 and variance 25 the other $15 \%$ of the time; and

4. Cauchy with median 0 and scale parameter 1.

Finally, after generating the $\eta_{\mathrm{t}}$, the $\varepsilon_{\mathrm{t}}$ values are created as $\varepsilon_{\mathrm{t}}=\rho \varepsilon_{\mathrm{t}-1}+\eta_{\mathrm{t}}$ where $\varepsilon_{0}=\frac{\eta_{0}}{1-\rho^{2}}$ and $\eta_{0}$ is an initial draw from the disturbance distribution. The values of $\rho$ used were $0.0,0.1$, $0.3,0.5,0.7$ and 0.9 .

The disturbances were generated independently of the explanatory variables. All random numbers were generated using IMSL subroutines and the simulation was written in FORTRAN.

The parameter $\beta_{0}$ was set equal to zero (without loss of generality). To determine 
empirical levels of significance, the parameter $\beta_{1}$ is set equal to zero, the test of $\mathrm{H}_{0}: \beta_{1}=0$ is performed, and the number of rejections of the true hypothesis is recorded.

The sample size used was $T=20$. For each factor level combination in the experimental design, 10,000 Monte Carlo trials were used to evaluate estimates and assess levels of significance. (Each factor level combination is determined by the disturbance distribution, type of independent variable and the value of the autocorrelation coefficient for a total of 144 factor level combinations).

Estimation

$$
\text { Results }
$$

Table 1 shows mean square error (MSE) ratios for the estimates of the coefficient of the explanatory variable. The ratios are of the MSE of each estimation method to the MSE of the LAV estimator. MSE ratios less than one favor each of the estimator types over LAV; MSE ratios greater than one favor LAV. These are medians of the results over the four error distributions (Cauchy, Laplace, Contaminated Normal, Normal). Each of the six explanatory variable types is listed in a separate panel of the table. Panels A, B and C are for autoregressive explanatory variables with Lambda $=0.0,0.4$ and 0.8 respectively.

For example, in Panel A the explanatory variable is autoregressive with Lambda $=0.0$ (that is, a normally distributed explanatory variable). The MSE ratio of LAVPW to LAV when Rho $=0.0$ is 1.01. Thus, LAV is favored over LAVPW (barely) in this instance. However, little is lost by performing the correction for autocorrelation. For the autoregressive independent variable, this is true in all cases when Rho $=0.0$. Although LAV is never unfavorable, there is often little or no difference, so the option to always correct for autocorrelation results in little loss in estimator efficiency. When the explanatory variable is autoregressive, there is little difference in whether the LAVPW or LAVCO correction is used. The LAVLAG alternative results in a larger loss in efficiency when Rho is small, for example the MSE ratio of LAVLAG to LAV is 1.05. As Rho increases, the relative efficiency of LAVLAG to LAV increases, but not as quickly as LAVPW or LAVCO when Lambda is 0.0 or 0.4 . However, when Rho is large and Lambda is 0.8 , the LAVLAG alternative results in greater efficiency than LAV and, in fact, greater efficiency than the other alternatives.

When the independent variable follows a stochastic trend (Panels D and E) it is also true that little is lost by performing the correction for autocorrelation. In this case, however, LAVPW is slightly better than LAVCO. The LAVLAG alternative shows a larger loss in efficiency when Rho is small than in the autoregressive case. For example the MSE ratio of LAVLAG to LAV is 1.16 for Lambda $=0.4$ and 1.07 for Lambda $=0.8$. As Rho increases, the relative efficiency of LAVLAG to LAV increases faster than LAVPW and the LAVLAG alternative soon provides greater efficiency than LAV and greater efficiency than the other alternatives.

The results for the fixed trend are similar to those for the stochastic trend, except that the LAVCO method fails miserably once Rho reaches 0.5 . The LAVLAG MSE ratio is 1.2 when Rho is zero, but this approach recovers quickly and is more efficient than any of the other approaches when Rho is 0.3 or greater. The primary conclusion from examination of MSEs is to avoid the LAVCO correction. A secondary conclusion is that LAVLAG compares favorably to LAVPW.

\section{Hypothesis Testing}

Empirical significance levels of the test for coefficient significance were examined. Due to the poor estimation performance of the LAVCO method, that procedure is eliminated from consideration. All tests were performed using a nominal level of 0.05 , thus, it is desirable to have the resulting empirical level close to this value. As a result, for purposes of this analysis a test is considered well-behaved if the empirical level is 0.06 or less.

Table 2 shows the number of times each method had an empirical significance level of 0.06 or less. Tests with larger numbers in Table 2 are viewed as more reliable because they do not overly reject true null hypotheses. The LR2, W2, LR1, LR3 and LM tests (in that order) had the highest total incidences of empirical levels that were at or below 0.06 over all the experimental design points. 
Table 1: MSE Ratios for the Estimates of the Coefficient of the Explanatory Variable

Panel A: Autoregressive with Lambda $=0.0$

\begin{tabular}{|c|c|c|c|c|c|c|}
\cline { 2 - 7 } \multicolumn{1}{c|}{} & \multicolumn{7}{c|}{ Rho } & 0.7 & 0.9 \\
\cline { 2 - 7 } \multicolumn{1}{c|}{} & 0.0 & 0.1 & 0.3 & 0.5 & 0.71 & 0.46 \\
\hline LAVPW & 1.01 & 1.00 & 0.91 & 0.77 & 0.61 & 0.46 \\
\hline LAVCO & 1.00 & 0.98 & 0.90 & 0.76 & 0.60 & 0.51 \\
\hline LAVLAG & 1.05 & 1.04 & 0.99 & 0.86 & 0.70 & \\
\hline
\end{tabular}

Panel B: Autoregressive with Lambda $=0.4$

\begin{tabular}{|c|c|c|c|c|c|c|}
\cline { 2 - 7 } \multicolumn{1}{c|}{} & \multicolumn{7}{c|}{ Rho } & 0.7 & 0.9 \\
\cline { 2 - 7 } \multicolumn{1}{c|}{} & 0.0 & 0.1 & 0.3 & 0.5 & 0.67 & 0.48 \\
\hline LAVPW & 1.02 & 0.97 & 0.92 & 0.81 & 0.67 & 0.48 \\
\hline LAVCO & 1.01 & 0.99 & 0.91 & 0.81 & 0.66 & 0.50 \\
\hline LAVLAG & 1.05 & 1.02 & 0.92 & 0.82 & 0.68 & \\
\hline
\end{tabular}

Panel C: Autoregressive with Lambda $=0.8$

\begin{tabular}{|c|c|c|c|c|c|c|}
\hline & \multicolumn{6}{|c|}{ Rho } \\
\hline & 0.0 & 0.1 & 0.3 & 0.5 & 0.7 & 0.9 \\
\hline LAVPW & 1.01 & 1.00 & 0.93 & 0.80 & 0.65 & 0.48 \\
\hline LAVCO & 1.01 & 1.00 & 0.92 & 0.81 & 0.66 & 0.48 \\
\hline LAVLAG & 1.07 & 1.01 & 0.87 & 0.71 & 0.53 & 0.37 \\
\hline
\end{tabular}

Panel D: Stochastic Trend with Lambda $=0.4$

\begin{tabular}{|c|c|c|c|c|c|c|}
\cline { 2 - 7 } \multicolumn{1}{c|}{} & \multicolumn{7}{c|}{ Rho } & 0.7 & 0.9 \\
\cline { 2 - 7 } \multicolumn{1}{c|}{} & 0.0 & 0.1 & 0.3 & 0.5 & 0.7 & 0.76 \\
\hline LAVPW & 1.00 & 1.01 & 0.96 & 0.86 & 0.76 & 0.84 \\
\hline LAVCO & 1.05 & 1.04 & 1.01 & 0.92 & 0.79 & 0.84 \\
\hline LAVLAG & 1.16 & 1.07 & 0.90 & 0.70 & 0.51 & 0.35 \\
\hline
\end{tabular}

Panel E: Stochastic Trend with Lambda $=0.8$

\begin{tabular}{|c|c|c|c|c|c|c|}
\cline { 2 - 7 } \multicolumn{1}{c|}{} & \multicolumn{7}{c|}{ Rho } & 0.7 & 0.9 \\
\cline { 2 - 7 } \multicolumn{1}{c|}{} & 0.0 & 0.1 & 0.3 & 0.5 & 0.65 & 0.48 \\
\hline LAVPW & 1.01 & 1.00 & 0.93 & 0.80 & 0.65 & 0.48 \\
\hline LAVCO & 1.01 & 1.00 & 0.92 & 0.81 & 0.66 & 0.37 \\
\hline LAVLAG & 1.07 & 1.01 & 0.87 & 0.71 & 0.53 & \\
\hline
\end{tabular}

Panel F: Linear Trend

\begin{tabular}{|c|c|c|c|c|c|c|}
\cline { 2 - 7 } \multicolumn{1}{c|}{} & \multicolumn{7}{c|}{ Rho } \\
\cline { 2 - 7 } \multicolumn{1}{c|}{} & 0.0 & 0.1 & 0.3 & 0.5 & 0.7 & 0.9 \\
\hline LAVPW & 1.01 & 1.01 & 0.99 & 0.94 & 0.88 & 0.88 \\
\hline LAVCO & 1.06 & 1.08 & 5.88 & 1351 & 1952 & 3455 \\
\hline LAVLAG & 1.20 & 1.10 & 0.92 & 0.73 & 0.56 & 0.45 \\
\hline
\end{tabular}

Notes: The ratios are of the MSE of each result to the MSE of the LAV estimator. MSE ratios less than one favor each of the estimator types over LAV; MSE ratios greater than one favor LAV. These are medians of the results over four error distributions. Each of the six explanatory variable types is listed in a separate panel of the table. 
Considering estimation procedures, the LAVLAG procedure had the most instances overall, 668, at or below 0.06. Combinations of test and estimation procedure that have the largest number of empirical significance levels at or below 0.06 are (in order): LAVLAG/LR1, LAVLAG/LR3, LAVLAG/W1 and LAVLAG/LR2. Note that LAVPW does not perform particularly well. LAVPW is the autocorrelation correction procedure typically recommended in previous studies. Also, LR3 is the test used in many previous studies, but LR1 or LR2 could be viewed as preferred in this study. This is consistent with the findings of Dielman (2006) in models without autocorrelation.

Table 3 provides detail on specific empirical levels of significance for estimation method/test combinations for selected values of the autocorrelation coefficient, Rho (panels in the table correspond to Rho $=0.0,0.1,0.3,0.5$, $0.7,0.9)$. The values in the table represent the median percentage of rejections for estimation method/test combinations with median taken over the four error distributions and over the six explanatory variable types. In the first panel of the table, for example, empirical levels of significance for $\mathrm{Rho}=0.0$ are shown .

The LAV method had empirical significance level of 0.06 or less for several of the tests: W2, LM, LR1, LR2, and LR3. The level for LAVPW was 0.06 or less for W2 and LR2. The LAVLAG method had level of 0.06 or less for W1, W3, LR1, LR2, LR3 and LR4.

When autocorrelation is at a moderate level of 0.5 , there are two combinations with empirical level of significance below 0.06: LAVLAG/LR1 and LAVLAG/LR3. All levels for LAV and LAVPW are above 0.06 and are similar for these two methods, even though LAVPW supposedly corrects for autocorrelation.

When Rho is 0.9 (a high level of autocorrelation), there are no cases when the empirical level of significance is below 0.06 . The closest values are 0.09 for LAVLAG/W1, LAVLAG/LR1 and LAVLAG/LR3. Note that the LAVPW method, one of the traditional corrections for autocorrelation, had very high empirical levels in a case when it might be expected to perform well. The levels are better than the uncorrected LAV, but still very high.

Table 2: Number of Times Each Method Had Empirical Significance Level of 0.06 or Less

\begin{tabular}{|c|c|c|c|c|c|c|c|c|c|c|}
\hline \multirow[b]{2}{*}{ Method } & \multicolumn{9}{|c|}{ Test } & \multirow[b]{2}{*}{ Totals } \\
\hline & W1 & W2 & W3 & W4 & LM & LR1 & LR2 & LR3 & LR4 & \\
\hline LAV & 21 & 85 & 17 & 9 & 67 & 56 & 84 & 46 & 24 & 409 \\
\hline LAVPW & 0 & 91 & 0 & 0 & 22 & 1 & 74 & 1 & 1 & 190 \\
\hline LAVLAG & 101 & 45 & 76 & 18 & 43 & 107 & 92 & 101 & 85 & 668 \\
\hline Totals & 122 & 221 & 93 & 27 & 132 & 164 & 250 & 148 & 110 & \\
\hline
\end{tabular}


Table 3: Empirical Levels of Significance (Proportion of Rejections) for Estimation Method/Test Combination for Selected Values of the Autocorrelation Coefficient, Rho

\begin{tabular}{|c|c|c|c|c|c|c|c|c|c|c|}
\hline \multirow[b]{2}{*}{ Rho } & \multirow[b]{2}{*}{ Method } & \multicolumn{9}{|c|}{ Test } \\
\hline & & W1 & W2 & W3 & W4 & $\mathrm{LM}$ & LR1 & LR2 & LR3 & LR4 \\
\hline \multirow{3}{*}{0} & LAV & 0.08 & 0.03 & 0.09 & 0.10 & 0.05 & 0.06 & 0.03 & 0.06 & 0.07 \\
\hline & LAVPW & 0.10 & 0.05 & 0.12 & 0.13 & 0.08 & 0.10 & 0.06 & 0.10 & 0.11 \\
\hline & LAVLAG & 0.05 & 0.07 & 0.06 & 0.08 & 0.12 & 0.05 & 0.05 & 0.05 & 0.06 \\
\hline \multirow{3}{*}{0.1} & LAV & 0.09 & 0.04 & 0.10 & 0.12 & 0.07 & 0.07 & 0.04 & 0.08 & 0.09 \\
\hline & LAVPW & 0.11 & 0.05 & 0.12 & 0.14 & 0.08 & 0.10 & 0.06 & 0.11 & 0.12 \\
\hline & LAVLAG & 0.05 & 0.07 & 0.06 & 0.08 & 0.12 & 0.05 & 0.06 & 0.05 & 0.06 \\
\hline \multirow{3}{*}{0.3} & LAV & 0.13 & 0.06 & 0.14 & 0.16 & 0.10 & 0.11 & 0.07 & 0.12 & 0.13 \\
\hline & LAVPW & 0.14 & 0.06 & 0.15 & 0.17 & 0.09 & 0.12 & 0.08 & 0.13 & 0.14 \\
\hline & LAVLAG & 0.06 & 0.07 & 0.06 & 0.08 & 0.13 & 0.05 & 0.06 & 0.06 & 0.07 \\
\hline \multirow{3}{*}{0.5} & LAV & 0.17 & 0.10 & 0.19 & 0.21 & 0.15 & 0.17 & 0.12 & 0.17 & 0.19 \\
\hline & LAVPW & 0.16 & 0.09 & 0.17 & 0.20 & 0.11 & 0.15 & 0.10 & 0.15 & 0.17 \\
\hline & LAVLAG & 0.07 & 0.08 & 0.07 & 0.09 & 0.14 & 0.06 & 0.07 & 0.06 & 0.07 \\
\hline \multirow{3}{*}{0.7} & LAV & 0.25 & 0.16 & 0.27 & 0.30 & 0.22 & 0.25 & 0.20 & 0.26 & 0.28 \\
\hline & LAVPW & 0.19 & 0.11 & 0.20 & 0.23 & 0.13 & 0.18 & 0.13 & 0.19 & 0.20 \\
\hline & LAVLAG & 0.08 & 0.09 & 0.08 & 0.10 & 0.15 & 0.07 & 0.08 & 0.08 & 0.09 \\
\hline \multirow{3}{*}{0.9} & LAV & 0.35 & 0.26 & 0.37 & 0.40 & 0.32 & 0.37 & 0.31 & 0.38 & 0.40 \\
\hline & LAVPW & 0.25 & 0.17 & 0.26 & 0.29 & 0.15 & 0.25 & 0.20 & 0.26 & 0.27 \\
\hline & LAVLAG & 0.09 & 0.11 & 0.10 & 0.12 & 0.18 & 0.09 & 0.10 & 0.09 & 0.11 \\
\hline
\end{tabular}

Note: These are medians of the results over the four error distributions and over the six explanatory variable types. 


\section{Conclusion}

The following conclusions are derived from the simulation study. Regarding estimation:

1. The LAVCO correction should be avoided due to possible extreme loss in efficiency.

2. The option to always correct for autocorrelation using the LAVPW correction never results in much efficiency loss.

3. Adding a lagged dependent variable rather than using the LAVPW correction is a viable option. The LAVLAG alternative typically results in a larger loss in efficiency than LAVPW when there is little autocorrelation, but an increase in efficiency when autocorrelation is more severe.

For hypothesis testing, the LAVLAG method had empirical levels of significance that were acceptable more often than LAVPW so is preferred in this sense. Both LAVPW and LAVLAG provide better protection against type one errors than LAV. However, the empirical levels of both are still high in some cases.

When estimating a regression with independent disturbances, Dielman and Rose (1995a, 2002) compared bootstrap tests to traditional tests in a LAV regression with independent errors and found that the bootstrap tests were generally competitive with LR tests that also perform well when disturbances are independent. It would be prudent to examine a bootstrap test in the context of autocorrelated errors as well; however, care must be taken in designing the bootstrap resampling process to preserve the autocorrelation structure.

\section{References}

Bassett, G., \& Koenker, R. (1978). Asymptotic theory of least absolute error regressions. Journal of the American Statistical Association, 73, 618-622.

Cochrane, D., \& Orcutt, G. (1949). Application of least squares regression to relationships containing autocorrelated error terms. Journal of the American Statistical Association, 44, 32-61.
Dielman, T. (2005). Least absolute value regression: Recent contributions. Journal of Statistical Computation and Simulation, 75, 263-286.

Dielman, T. (2006). Variance estimates and hypothesis tests in least absolute value regression. Journal of Statistical Computation and Simulation, 76, 103-114.

Dielman, T., \& Pfaffenberger, R. (1984). Small sample properties of estimators in the autocorrelated error model: A review and some additional simulations. Statistical Papers/Statistiche Hefte, 30, 163-183.

Dielman, T., \& Pfaffenberger, R. (1990). Tests of linear hypotheses in LAV regression. Communications in Statistics Simulation and Computation, 19, 1179-1199.

Dielman, T., \& Pfaffenberger, R. (1992). A further comparison of tests of hypotheses in LAV regression, Computational Statistics and Data Analysis, 14, 375-384.

Dielman, T., \& Rose, E. (1994a). Estimation in least absolute value regression with autocorrelated errors. Journal of Statistical Computation and Simulation, 50, 29-43.

Dielman, T., \& Rose, E. (1994b). Forecasting in least absolute value regression with autocorrelated errors: a small-sample study. International Journal of Forecasting, 10, 539547.

Dielman, T., \& Rose, E. (1995a). A bootstrap approach to hypothesis testing in least absolute value regression. Computational Statistics and Data Analysis, 20, 119-130.

Dielman, T., \& Rose, E. (1995b). Estimation after pre-testing in least absolute value regression with autocorrelated errors. Journal of Business and Management, 2, 74-95.

Dielman, T., \& Rose, E. (1996). A note on hypothesis testing in LAV multiple regression: A small sample comparison. Computational Statistics and Data Analysis, 21, 463-470.

Dielman, T., \& Rose, E. (1997). Estimation and testing in least absolute value regression with serially correlated disturbances. Annals of Operations Research, 74, 239-257. 


\section{LAV REGRESSION WITH AUTOCORRELATED ERRORS: IS CORRECTION HELPFUL?}

Dielman, T., \& Rose, E. (2002). Bootstrap versus traditional hypothesis testing procedures for coefficients in least absolute value regression. Journal of Statistical Computation and Simulation, 72, 665-675.

Koenker, R. (1987). A comparison of asymptotic testing methods for $\mathrm{L}_{1}$-regression. In: Y. Dodge (Ed.), Statistical data analysis based on the $L_{1}$-norm and related methods, 287-295. Amsterdam: North-Holland.

Koenker, R., \& Bassett, G. (1982). Tests of linear hypotheses and $L_{l}$ estimation. Econometrica, 50, 1577-1583.

Maeshiro, A. (1979). On the retention of the first observation in serial correlation adjustment of regression models. International Economic Review, 20, 259-265.

Mizon, G. (1995). A simple message for autocorrelation correctors: Don't. Journal of Econometrics, 69, 267-288.

McKean, J., \& Schrader, R. (1984). A comparison of methods for studentizing the sample median. Communications in Statistics Simulation and Computation, 13, 751-773.
Park, R., \& Mitchell, G. (1980). Estimating the autocorrelated error model with trended data. Journal of Econometrics, 13, 185201.

Prais, S., \& Winsten, C. (1954). Trend estimators and serial correlation. Cowles Commission Discussion Paper: Stat. No. 383, Chicago.

Sheather, S. (1987). Assessing the accuracy of the sample median: Estimated standard errors versus interpolated confidence intervals. In: Y. Dodge (Ed.), Statistical data analysis based on the $L_{1}$-norm and related methods, 203-215. Amsterdam: North-Holland.

Stangenhaus, G. (1987). Bootstrap and inference procedures for $L_{1}$ regression. In: Y. Dodge (Ed.) Statistical data analysis based on the $L_{1}$-norm and related methods, 323-332. Amsterdam: North-Holland.

Weiss, A. (1990). Least absolute error estimation in the presence of serial correlation. Journal of Econometrics, 44, 127-158. 